\title{
Limiting Damage to 2D Materials during Focused Ion Beam Processing
}

Andersen, Anton Bay; Shivayogimath, Abhay; Booth, Tim ; Kadkhodazadeh, Shima; Hansen, Thomas Willum

Published in:

Physica Status Solidi (b)

Link to article, DOI:

$10.1002 /$ pssb.202000318

Publication date:

2020

Document Version

Peer reviewed version

Link back to DTU Orbit

Citation (APA):

Andersen, A. B., Shivayogimath, A., Booth, T., Kadkhodazadeh, S., \& Hansen, T. W. (2020). Limiting Damage to 2D Materials during Focused lon Beam Processing. Physica Status Solidi (b), 257(12), [2000318].

https://doi.org/10.1002/pssb.202000318

\section{General rights}

Copyright and moral rights for the publications made accessible in the public portal are retained by the authors and/or other copyright owners and it is a condition of accessing publications that users recognise and abide by the legal requirements associated with these rights.

- Users may download and print one copy of any publication from the public portal for the purpose of private study or research.

- You may not further distribute the material or use it for any profit-making activity or commercial gain

- You may freely distribute the URL identifying the publication in the public portal

If you believe that this document breaches copyright please contact us providing details, and we will remove access to the work immediately and investigate your claim. 


\section{Limiting damage to 2D materials during focused ion beam processing}

Anton Bay Andersen, Abhay Shivayogimath, Tim Booth, Shima Kadkhodazadeh, Thomas Willum Hansen*

Keywords: 2D Materials, $\mathrm{MoS}_{2}$, FIB, Surface Damage

2D materials are by definition just, a few atomic layers thick. They are therefore ideal samples for transmission electron microscopy, in the plan view geometry. However, 2D materials are typically placed or grown on substrates, which in some cases requires analysis to be performed on cross sections. In this case focused ion beam preparation is often the technique of choice for producing thin lamellae, but surface to the 2D material damage during ion imaging and milling must be mitigated. Here, it is demonstrated that the typically applied electron beam assisted deposition of platinum and carbon prior to milling does not provide sufficient protection and results in significant damage. Instead, it is found that arc evaporated carbon -deposited with a standard carbon coater designed for SEM samples- can provide sufficient protection, enabling cross-sectional analysis without detectable damage to monolayer or bilayer samples subsequently prepared by standard focused ion beam preparation procedures.

\section{Introduction}

Whether grown by chemical vapor deposition (CVD), exfoliated, transferred or in a final device, 2D materials are often supported by a substrate. For these structures, cross sectional samples offer a direct way of investigating the number of layers, the morphology, the crystal structure and interfaces using (scanning) transmission electron microscopy ((S)TEM). Despite their inherent electron transparency in plan-view, (S)TEM investigation of 2D materials in cross-section requires extensive thinning and polishing. Focused ion beam (FIB) milling and lift out of micron size lamellae to TEM compatible grids inside the FIB chamber offers many advantages for the preparation of samples for substrate-bound structures of 2D materials on-a substrate. It allows for

This article has been accepted for publication and undergone full peer review but has not been through the copyediting, typesetting, pagination and proofreading process, which may lead to differences between this version and the Version of Record. Please cite this article as doi: $10.1002 /$ pssb.202000318. 


\section{WILEY-VCH}

the rapid preparation of samples from specific selected regions and is highly versatile in terms of the types of materials that can be prepared. Such versatility has required efforts to understand and reduce FIB induced damage by developing specific milling recipes. ${ }^{[1-5]}$ As an example, FIB can be used to prepare structures after growth or after patterning/contacting for (S)TEM analysis. ${ }^{[6-8]}$ Surface damage introduced during FIB preparation can often be limited to a depth range of few nanometers by either depositing protective layers prior to milling or by adjusting the milling parameters. ${ }^{[9-15]}$ This is sufficient in cases, where the surface atoms are not of explicit interest, such as examination of a metal contact to a $2 \mathrm{D}$ material. ${ }^{[7]}$ In cases where the $2 \mathrm{D}$ material is the very top layer, as for CVD grown 2D materials, hardly any damage from the FIB milling can be tolerated. This work shows that arc evaporated carbon -performed with a standard carbon coater designed for SEM samples- provides sufficient protection, enabling cross-sectional analysis of 2D materials with no detectable damage to monolayer or bilayer samples. Furthermore, it is shown that electron beam assisted deposition of platinum produces surface damage to gold surfaces with a depth greater than the typical thickness of typical 2D materials.

\section{Results and discussion}

Cross section of five different sample types are studied by (S)TEM here. All five lamellae were made with the same FIB preparation procedure. A full description of used materials and procedures all samples can be found in the experimental section and a sketch of the layered structure is shown in figure S1. We note here that electron beam deposited platinum is not pure platinum, but a mixture of amorphous carbon and platinum particles with a high carbon content. ${ }^{[16-17]}$ Therefore, the major difference between samples with arc evaporated carbon and electron beam deposited platinum top layers is not the is not the material that constitutes the layer. It is the deposition technique used to deposit the layer, whether this is by condensation of evaporated carbon during arc-discharge, or by electron induced CVD of a metal organic precursor. 


\section{WILEY-VCH}

Figure 1 shows a comparison of high resolution transmission electron microscope (HRTEM) images of the pure gold surfaces of sample 1 and 2 . They are used to compare the damage done by the two methods without added effect of a 2D material layer. The surface of the gold samples was known to be the $\{111\}$ plane and both samples were milled along $<111>$ direction. The orientation of the milling pattern relative to the crystal structure was however unknown, as the rotation of the crystal around the $\langle 111>$ orientation was unknown, so the available zone axis perpendicular to the $<111>$ direction, within the tilting range of the sample holder, was different for the two samples. For sample 1, coated with electron beam deposited platinum, this was a $<110>$ zone axis and for sample 2, coated with arc evaporated carbon, it was a $<112>$ zone axis. Both samples show clear crystalline structure in the gold. The intensity profiles of the two images, summed along the horizontal direction, are shown to the right. In Figure 1a) the lattice fringes and mass contrast transition smoothly from the substrate to the protection layer over a distance of approximately $1 \mathrm{~nm}$. On Figure 1b) the transition of lattice fringes and mass contrast is sharper and transitions over a distance of approximately $0.2 \mathrm{~nm}$. This difference cannot be attributed to a difference in defocus (1)

resulting in blurring, as inspection of the TEM image in a) reveals a corrugated surface rather than a gradual transition. The regions shown here are representative cutouts of larger images, to show the necessary detail. These larger images can be seen in Figure S2.

The surface roughness is 4-5 atomic layers for, the sample coated with electron deposited platinum, but around one atomic layer for the sample coated with evaporated carbon. Since the deposition of protective layers is not expected to flatten the surface, the greater roughness of sample 1 must be introduced during deposition of the protective layers.

Figure 2 a) shows an optical image of a monolayer $\mathrm{MoS}_{2}$ flake, used to make sample 3, after exfoliation. The flake is expected to be of monolayer thickness prior to processing as the lamella was taken from a region of minimal contrast, see optical image of larger region in Figure S3. The 


\section{WILEY-VCH}

region where the lamella was extracted from based on secondary electron images acquired during FIB processing is shown in Figure S3. Figure 2 b) shows a cross sectional high angle annular dark field (HAADF)-STEM image of the exfoliated monolayer $\mathrm{MoS}_{2}$ on the sputtered gold surface with evaporated carbon as the first protection layer. Figure $2 \mathrm{c}$ ) shows a vertically averaged line scan of the image intensity in the blue box in $2 \mathrm{~b}$ ) and an insert of the expected atomic structure of $\mathrm{MoS}_{2}$ in the $<112>$ orientation. ${ }^{[18]}$ The measured inter column distance of $0.16 \mathrm{~nm}$ corresponds well to the crystal structure of $\mathrm{MoS}_{2}$. The sample was aligned with respect to the silicon substrate crystal, see experimental details for the full sample structure, and therefore only the shown $\langle 110\rangle$ orientation of the $\mathrm{MoS}_{2}$ layer could be found. The good correspondence of the measured and expected atomic structure suggests that the thickness of evaporated carbon is sufficient protection for a single

monolayer of $\mathrm{MoS}_{2}$. By comparison, sputter coating exfoliated multilayer $\mathrm{MoS}_{2}$ with gold produces damage extending several layers deep, see figure S5. Figure 2 d) shows a HAADF-STEM of image multilayer $\mathrm{MoS}_{2}$ as grown by CVD on a monocrystalline, (within the feature size of a TEM lamella), gold substrate protected with evaporated carbon. As the 2D material are grown epitaxially on the gold (111) by this process, it is possible to controllably tilt the $\mathrm{MoS}_{2}$ layers to a $<110>$ zone axis by tilting the gold substrate to a $<110>$ zone axis. ${ }^{[21]}$ Figure 2 e) shows vertically averaged line scans over the sulfur (red box in d)) and molybdenum (blue box in d)) in red and blue respectively. The insert of the atomic structure of $\mathrm{MoS}_{2}$ shows good agreement between the inter column distance of $0.26 \mathrm{~nm}$ and the offset of the sulfur and molybdenum columns. This shows that evaporated carbon can also protect CVD grown $\mathrm{MoS}_{2}$ from structural damage during preparation of FIB lamellae to allow accurate characterization of the atomic structure. Cross sectional TEM results and comparison with simulations suggest similar results for CVD grown layers of $\mathrm{PtS}_{2}$, see S6. Both HAADF-STEM (Figure 2 b) and d)) images are cut out of larger images and have undergone scan noise correction and rolling background removal to highlight the position of the atomic 


\section{WILEY-VCH}

columns due to the large difference in atomic number between gold and sulfur and final smoothening to reduce shot noise. The full original images and overview images can be seen in S3. These results taken together suggest no structural damage of $\mathrm{MoS}_{2}$, detectable by imaging, when using evaporated carbon as a protection layer. A potentially more sensitive way to test for a measurable indication of damage is the electron energy-loss spectroscopy fine-structure for the sulfur atoms since they constitute the top part of the $\mathrm{MoS}_{2}$ layer. Therefore the sulfur $\mathrm{L}_{2,3}$ edges is examined. Figure 4 a) shows an HAADF-STEM image of CVD grown $\mathrm{MoS}_{2}$ monolayer on gold coated with evaporated carbon (sample 5). The red and blue lines indicate where the electron energy loss (EEL) spectra shown in Figure 2 b) were acquired. Note that the distance between these regions is approximately $1 \mathrm{~nm}$, which is larger than the width of a single $\mathrm{MoS}_{2}$ layer. This is done because the delocalization of the excitation would otherwise mean the EEL spectra would probe both the inner and outer sulfur layers. ${ }^{[19]}$ The background subtracted and normalized EELS signal from the inner (blue dots) and outer (red crosses) sulfur layers are seen to be similar within noise levels. The fine structure of bulk $\mathrm{MoS}_{2}$ (black line) (the clear pre peak at $165 \mathrm{eV}$ and the weaker second peak on top of the onset of the full edge at $175 \mathrm{eV}$ ) is recognizable in both the outer and inner sulfur layers. These two features are different from the amorphous sulfur reference spectra supplied by Gatan, which has a smoother onset of the edge beginning at $165 \mathrm{eV} .{ }^{[20]}$ The EEL spectra prior to background subtraction and normalization can be seen in Figure S4. Note that plural scattering correction has not been performed and thus a slight difference between the ratio of the two peaks is likely due to the sample not having the same thickness. Changes to the fine structure of the sulfur $\mathrm{L}_{2,3}$ were therefore not detectable in monolayer $\mathrm{MoS}_{2}$ between the outer and inner sulfur layer, and both are in agreement with a bulk $\mathrm{MoS}_{2}$ reference sample with respect to the relative locations of pre peak and edge onset.

\section{Conclusion}

We have examined pure gold surfaces, $\mathrm{MoS}_{2}$ multi and monolayers grown by CVD and exfoliated $\mathrm{MoS}_{2}$ monolayers in cross section, using TEM, STEM and STEM-EELS. We have shown that 


\section{WILEY-VCH}

evaporated carbon -in contrast to commonly employed electron beam deposited platinum- produces no detectable damage to pure gold surfaces nor $\mathrm{MoS}_{2}$ layers whether they are exfoliated or CVD grown. These results are important for the study of 2D material based devices and for post synthesis characterization of 2D materials. They also have important implications for FIB investigation and preparation of exposed surfaces in general. The methods described provide a simple to follow procedure using standard tools readily available in most laboratories equipped for SEM and TEM sample preparation, and can thus be readily applied by those working with FIB preparation and subsequent characterization of 2D materials, and sensitive exposed surfaces in general.

\section{Experimental Section}

Materials:

Sample 1 and 2 differ only by the protective layers deposited prior to FIB milling, and otherwise consist of annealed gold on a c-plane sapphire wafer with the gold surface being the $\{111\}$ plane. ${ }^{[21]}$ Sample 3 exfoliated $\mathrm{MoS}_{2}$ on gold and was made according to the methods described in. ${ }^{\text {[22] }}$ Sample 4 and 5 are CVD grown $\mathrm{MoS}_{2}$ and were made with the method described in. ${ }^{[21]}$ Chemically exfoliated $\mathrm{MoS}_{2}$ samples, used for electron energy loss spectroscopy (EELS) reference measurements, were made with the method described by Coleman et al. using 1-methyl-2pyrrolidinone (NMP) as the solvent. ${ }^{[23]}$

\section{Methods:}

All five samples were mounted on an aluminum SEM stub using Crystal Bond and the edges were painted with silver paint to minimize charging during SE imaging and FIB milling. The FIB milling of all five samples was carried out on a FEI-Helios dual beam system operated at 5-10kV and $0.17-5.5 \mathrm{nA}$ for the electron beam and $30 \mathrm{kV}$ and $28-20,000 \mathrm{pA}$ for the Ga+ beam. Prior to milling the region of interest on the samples was covered with $0.2-0.5 \mu \mathrm{m}$ electron beam deposited platinum and 2-3 $\mu \mathrm{m} \mathrm{Ga}+$ deposited platinum. During the final thinning steps the ion beam current was lowered stepwise from 280 to $26 \mathrm{pA}$ during the milling. The samples were then tilted \pm 5 


\section{WILEY-VCH}

degrees with respect to the ion beam and polished at $2 \mathrm{kV}$ and $6 \mathrm{pA}$ for $10 \mathrm{~min}$ on both sides.

Samples 2-5 were coated with carbon (Cressington Carbon 208, 60-90s, 50-100A) to protect the surface and subsequently sputter coated with gold (Quorum Q150T ES, 20mA, 10s) to further reduce charging during imaging.

TEM measurements were carried out using an image corrected FEI Titan E-Cell 80-300 ST TEM operated at 300kV. STEM and STEM-EELS measurements were carried out on an FEI Titan 80300 ST TEM equipped with CESCOR pre objective lens spherical aberration corrector and a tridem 865 GIF system operated at $300 \mathrm{kV}$.

\section{Supporting Information}

Supporting Information is available from the Wiley Online Library or from the author.

\section{Acknowledgements}

The authors declare no conflicts of interest.

\section{Acknowledgements}

A. B. A, T. B., A. S. and T. W. H acknowledge support from the Danish National Research

Foundation Center of Excellence for Nanostructured Graphene (CNG) (project DNRF103)

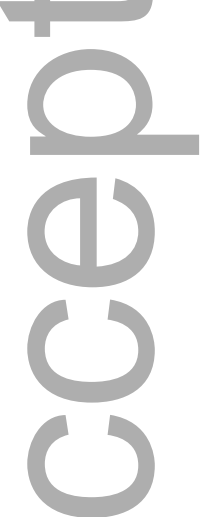

Received: ((will be filled in by the editorial staff))

Revised: ((will be filled in by the editorial staff)) Published online: ((will be filled in by the editorial staff))

References

[1] J. P. McCaffery, M.W. Phaneuf, L.D. Madsen, Ultramicroscopy, 2001, 87, 97

[2] S. Rubanov, P. R. Munroe, Journal of Microscopy, 2004, 214, 213

[3] S. Miroslava, S. Bernhard, Q. Ramasse, Ultramicroscopy, 2012, 114, 62

[4] L. Lan-Hsuan, Y. Chia-Hao, W. Chuan-Yu, L. Pei-Chin, H. Jih-Shang, Ultramicroscopy, 2019, 197,95 


\section{WILEY-VCH}

[5] L. Chen, H. Gerlinde, C. B. Lisa, A. Rainer, Ultramicroscopy, 2018, 187, 310

[6] L. Ang-Yu, Z. Hanyu, X. Jun, C. Chih-Piao, H. Yimo, C. Ming-Hui, C. Chia-Chin, Y. Chih-

Wen, W. Kung-Hwa, Y. Yiming, W. Yuan, S. Dimosthenis, N. Dennis, Y. Peidong, A. M. David, C.

Mei-Yin, Z. Xiang, L. Lain-Jong, Nature Nanotechnology, 2017, 12, 744

[7] W. Yan, C. K. Jong, J. W. Ryan, M. Jenny, S. Xiuju, Y. Jieun, Z. Fang, M. Andre, Y. J. Hu, C.

Manish, Nature, 2019, 568, 70

[8] L. Wang, I. Meric, P. Y. Huang, Q. Gao, Y. Gao, H. Tran, T. Taniguchi, K. Watanabe, L. M.

Campos, D. A. Muller, J. Guo, P. Kim, J. Hone, K. L. Shepard, C. R. Science, 2013, 342

[9] Y. Park, B. C. Park, S. Romankov, K. J. Park, J. H. Yoo, Y. B. Lee, J. M. Yang, Journal of

Microscopy, 2014, 255, 180

[10] S. Rubanov, P.R. Munroe, Material Letters, 2003, 225, 180

[11] M. Baram, W.D Kaplan, Journal of Mircroscopy, 2008, 232, 395

[12] N. I. Kato, Journal of Electron Microscopy, 2004, 53m, 451

[13] D. J. Barber, Ultramicroscopy, 1993, 52, 101

[14] B. W. Kempshall, L. A. Giannuzzi, B. I. Prenitzer, F. A. Stevie, S. X. Da, Jornal of Vacuum Science \& Technology B, 2002, 20, 286

[15] S. Hata, H. Sosiati, N. Kuwano, M. Itakura, T. Nakano and Y. Umakoshi, Journal of Electron Microscopy, 2006, 55, 23

[16] B. An, Y. Kwon, J. Oh, Y. Shin, J. Ju, C. Yang, Applied Microscopy, 2019, 49, 6

[17] W.Y. Kwong, W.Y. Zhang, ISSM 2005, IEEE International Symposium on Semiconductor Manufacturing, 2005, 469

[18] Y. Takeuchi and W. Nowacki, Schweizerische Mineralogische und Petrographische

Mitteilungen, 1964, 44, 105

[19] R. F. Egerton, Ultramicroscopy, 2007, 107, 575 


\section{WILEY-VCH}

[20] Gatan Inc, EELS atlas sulfur edge $\mathrm{L}_{2,3}$ spectrum, https://eels.info/atlas/sulfur, acsessed: April, 2020.

[21] A. Shivayogimath, J. D. Thomsen, D. M. A. Mackenzie, M. Geisler, R. Stan, A. J. Holt, M.

Bianchi, A. Crovetto, P. R. Whelan, A. Carvalho, A. H. C. Neto, P. Hofmann, N. Stenger, P.

Bøggild \& T. J. Booth, Nature communications, 2019, 10

[22] M. Velicky , G. E. Donnelly, W. R. Hendren, S. McFarland, D. Scullion, W. J. I. DeBenedetti,

G. C. Correa, Y.Han, A. J. Wain, M. A. Hines, D. A. Muller, K. S. Novoselov, H. D. Abrun, R. M.

Bowman, E. J. G. Santos, and F. Huang, ASC Nano, 2018, 12, 10463

[23] J. N. Coleman, M. Lotya1, A. O’Neill, S. D. Bergin, P. J. King, U. Khan, K. Young, A. G., S.

De, R. J. Smith, I. V. Shvets, S. K. Arora, G. Stanton, H. Kim, K. Lee, G. T. Kim, G. S. Duesberg,

T. Hallam, J. J. Boland, J. J. Wang, J. F. Donegan, J. C. Grunlan, G. Moriarty, A. Shmeliov, R. J.

Nicholls, J. M. Perkins, E. M. Grieveson, K. Theuwissen, D. W. McComb, P. D. Nellist, V.

Nicolosi, Science, 2011, 331, 568

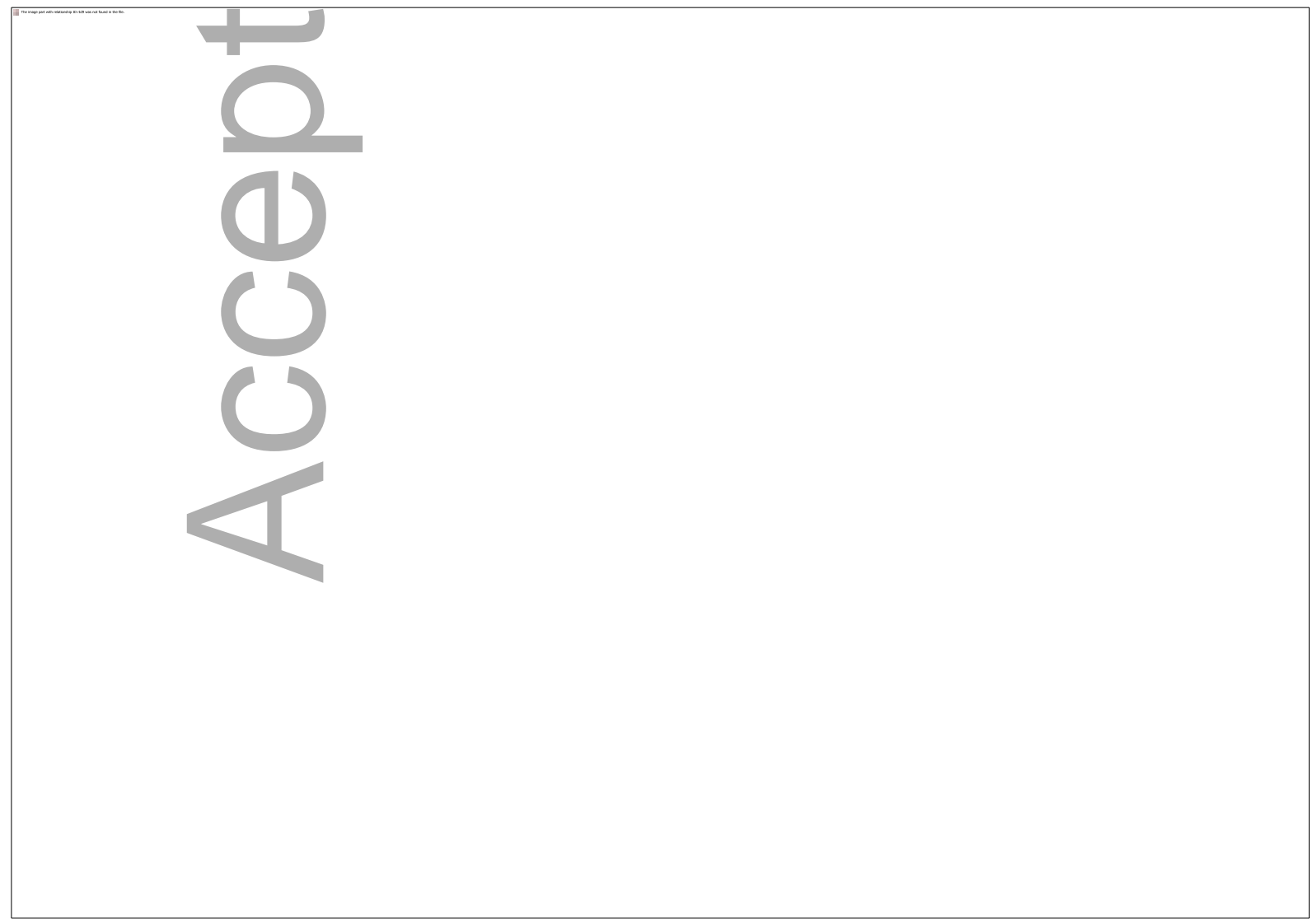

This article is protected by copyright. All rights reserved 


\section{WILEY-VCH}

Figure 1: Comparison of the surface damage introduced with and without protective carbon coating, seen from cross sectional HRTEM images of the gold surface of sample 1 at $\langle 110\rangle$ zone axis (a) and sample 2 at $<112>$ zone axis (b). Image intensity integrated horizontally with indication of final surface roughness using evaporated carbon layer (b) and with electron deposited platinum (a).

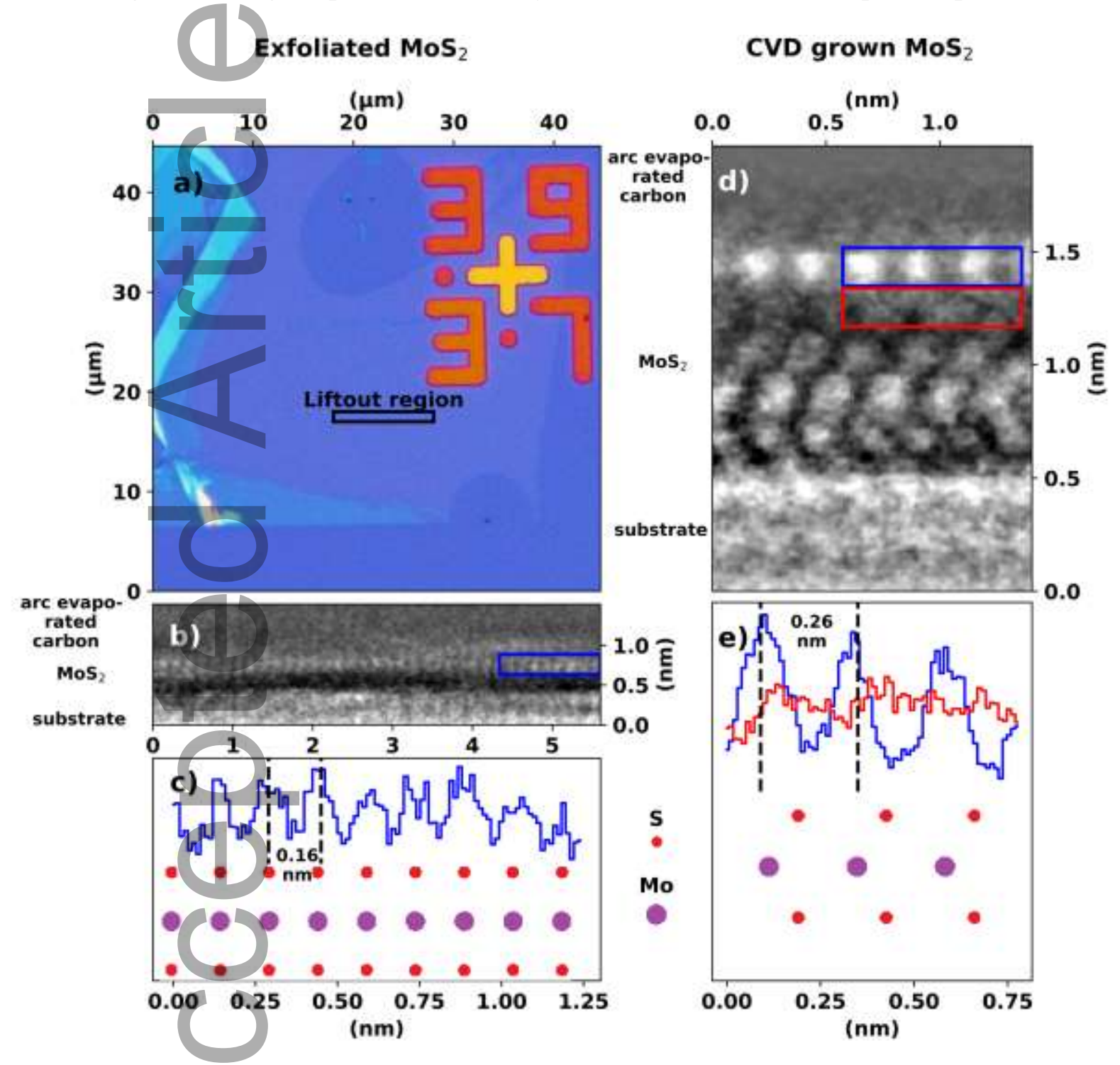

Figure 2: (a) Optical micrograph of exfoliated $\mathrm{MoS}_{2}$ (sample 3) showing color contrast corresponding to monolayer $\mathrm{MoS}_{2}$, prior to application of protective coatings, with indication of the region where the lamellae was extracted. (b) Cross sectional STEM-HAADF image of the surface of sample 3 showing monolayer $\mathrm{MoS}_{2}$ (c) Vertically averaged image intensity from the blue box in the STEM-HAADF image above with comparison of expected position of molybdenum atoms and peaks in image intensity. (d) Cross sectional STEM-HAADF image of bilayer CVD grown $\mathrm{MoS}_{2}$ 


\section{WILEY-VCH}

(sample 4). (e) Vertically averaged image intensity from the blue and red box in the STEMHAADF image above, shown as blue and red curves respectively, with comparison of image intensity and the expected position of the sulfur and molybdenum atoms.

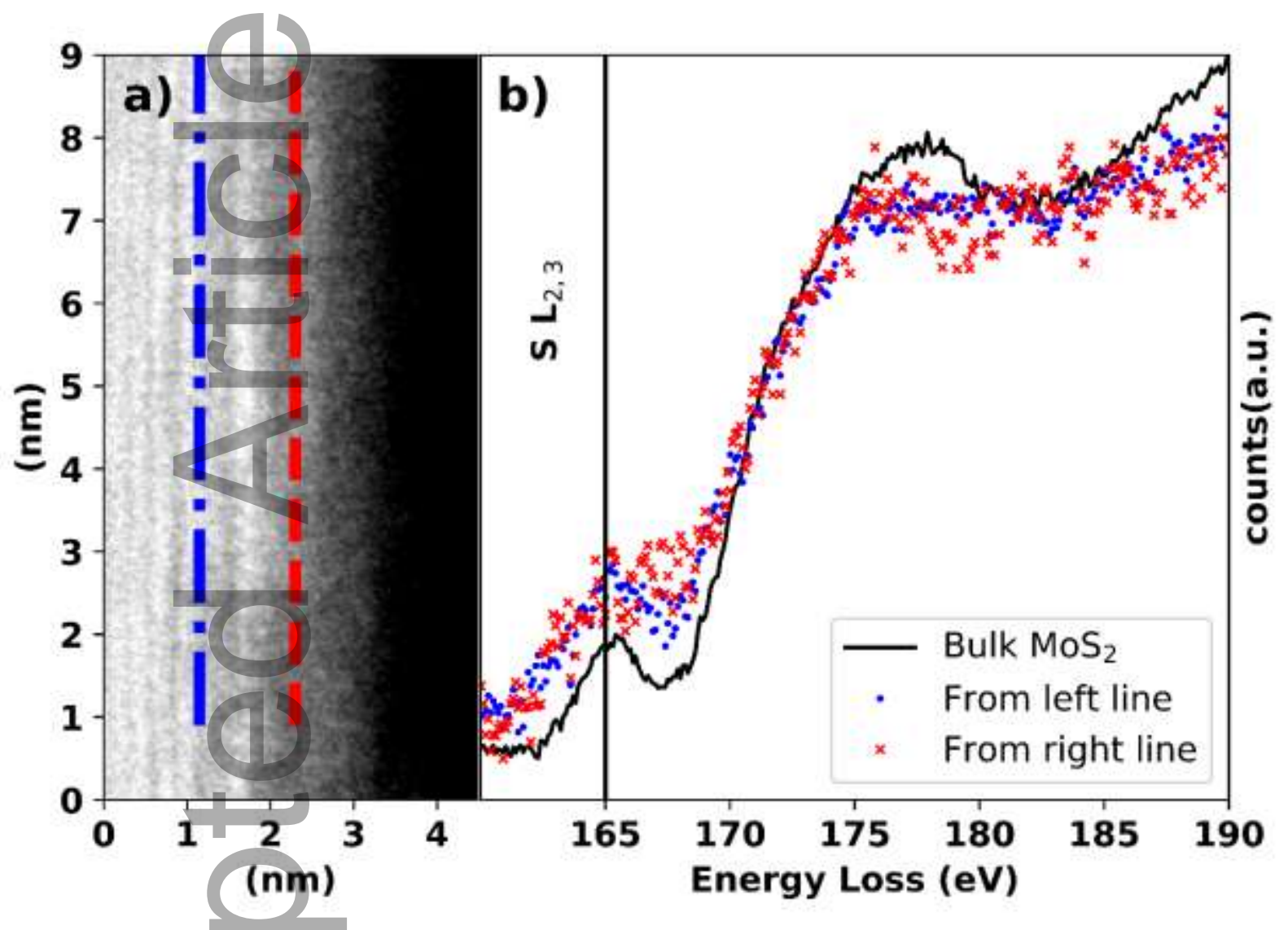

Figure 3: (a) Cross sectional STEM-DF image showing Monolayer $\mathrm{MoS}_{2}$ on the surface of sample

5. (b) Background subtracted, normalized and onset corrected EELS spectra acquired along the red and blue lines in the STEM-HAADF image shown as red and blue dots respectively and a reference spectrum (black line) from bulk $\mathrm{MoS}_{2}$ acquired with the same microscope parameters and with the bulk crystal oriented as the monolayer $\mathrm{MoS}_{2}$ shown to the left. The energy resolution, FWHM of zero loss peak, in the spectra is $1 \mathrm{eV}$

This article is protected by copyright. All rights reserved 
WILEY-VCH

Preparing exposed 2D materials or surfaces for cross-sectional investigations at the atomic level requires surface damage to be minimized at the atomic level. This work presents a simple procedure for elimination surface damage, measurable with TEM, STEM and EELS, during FIB preparation of lamella of exposed $\mathrm{MoS}_{2}$ on gold using evaporated carbon.

\section{Keyword: Surface Damage}

Anton Bay Andersen, Abhay Shivayogimath, Tim Booth, Shima Kadkhodazadeh, Thomas Willum

Hansen*

\section{Limiting damage to 2D materials during focused ion beam processing}

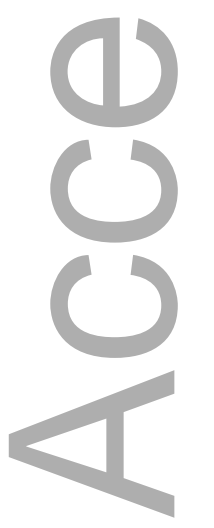

This article is protected by copyright. All rights reserved 
WILEY-VCH

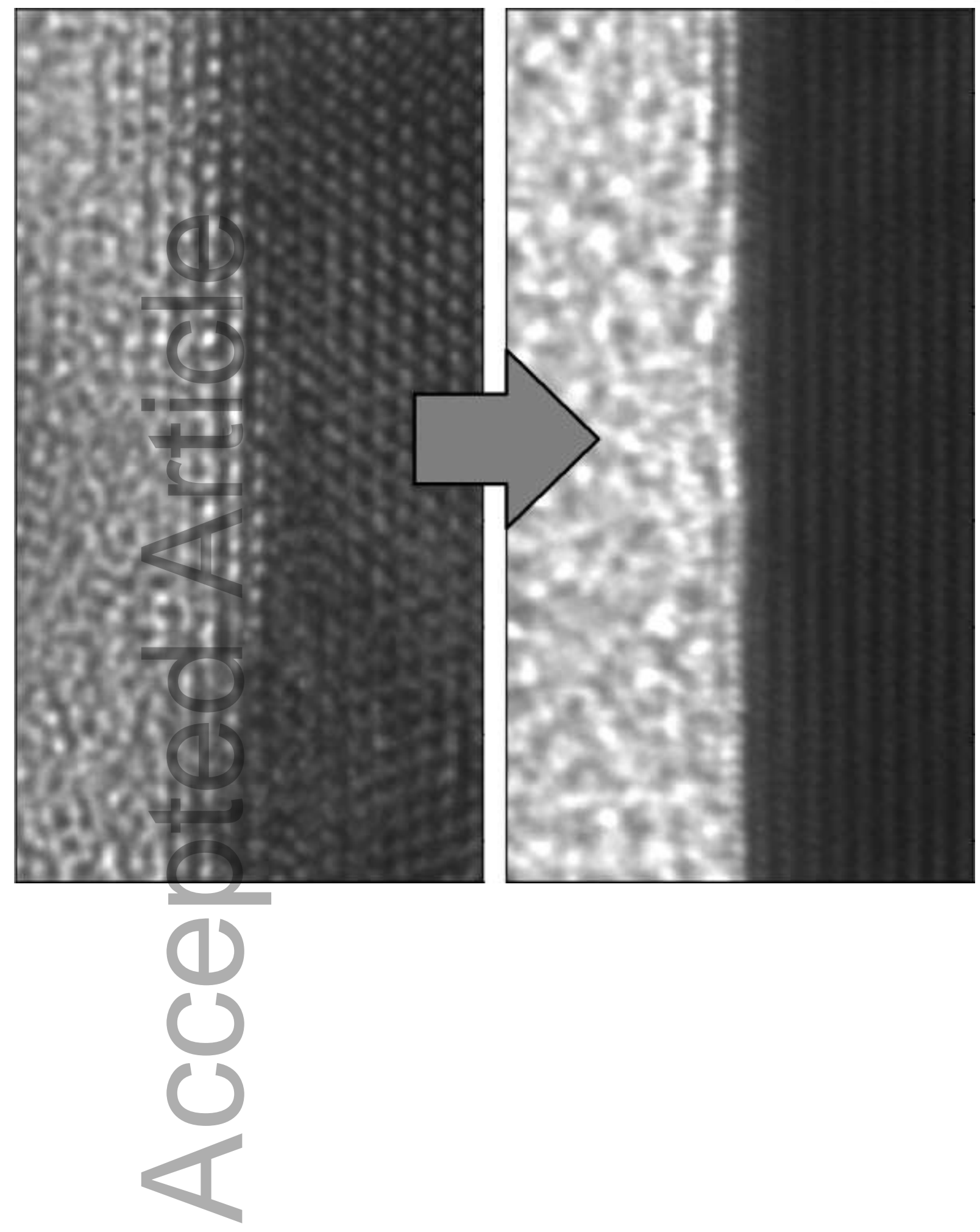

This article is protected by copyright. All rights reserved 